\title{
Câncer colorretal: a importância de um rastreio precoce
}

\author{
Colorectal câncer: the importance of early screening
}

Cáncer colorrectal: la importância de la detección precoz

Yasmin dos Santos Felisberto ${ }^{1 *}$, Cinara Dourado Pereira Correia Santos ${ }^{1}$, Poliana Terra Pires Ribeiro Coelho Caires ${ }^{1}$, Ana Carolina de Oliveira Bitencourt ${ }^{1}$, Ana Virgínia Figueira Dubois Mendes ${ }^{1}$, João Matheus Barbosa de Lima Pinho ${ }^{1}$, Rosy Aline Lopes de Oliveira ${ }^{1}$, Beatriz Tianeze de Castro ${ }^{1}$, Paula Mendes Ribeiro e Oliveira ${ }^{1}$, Julia Menezes Santos ${ }^{1}$.

\section{RESUMO}

Objetivo: Revisar e refletir acerca da importância da realização dos exames de rastreamento do Câncer colorretal (CCR). Revisão Bibliográfica: O CCR é uma neoplasia que acomete o intestino grosso, sendo responsável por números crescentes de óbitos nos países Ocidentais. Nesse cenário, temos que no Brasil, com exceção do câncer de pele não melanoma, essa neoplasia maligna apresenta incidência alta, sendo o segundo tipo de câncer mais prevalente nas mulheres e o terceiro mais prevalente nos homens. A maioria dos tumores são de causas esporádicas e podem ser evitados pela prevenção primária através de uma dieta equilibrada e prática regular de atividade física. Além disso, surge nesse contexto um destaque para a prevenção secundária, pois a utilização dos exames de rastreio e as campanhas voltadas para a identificação precoce são ferramentas essenciais para se evitar a progressão da doença na sequência adenomacarcinoma. Considerações finais: Apesar da elevada incidência no país, o CCR é uma enfermidade curável quando detectada nos estágios iniciais. Dessa forma, desenvolver novos estudos sobre os métodos de pesquisa, diagnóstico e rastreamento, buscar o aprimoramento profissional e estabelecer políticas públicas eficientes nas campanhas que abrangem a prevenção secundária são estratégias significativas no combate à essa doença silenciosa.

Palavras-chave: Câncer colorretal, Cólon, Reto, Rastreio.

\begin{abstract}
Objective: To review and reflect on the importance of performing colorectal cancer screening tests (CCR). Bibliographic review: CCR is a neoplasm that affects the large intestine, being responsible for increasing numbers of deaths in Western countries. In this scenario, in Brazil, with the exception of non-melanoma skin cancer, this malignant neoplasm has a high incidence, being the second most prevalent type of cancer in women and the third most prevalent in men. Most tumors are sporadic and can be prevented by primary prevention through a balanced diet and regular physical activity. In addition, in this context, secondary prevention is highlighted, since the use of screening tests and campaigns aimed at early identification are essential tools to prevent the progression of the disease following adenoma-carcinoma. Final considerations: Despite the high incidence in the country, CCR is a curable disease when detected in the early stages. Thus, developing new studies on research, diagnosis and screening methods, seeking professional improvement and establishing efficient public policies on efficiency that cover secondary prevention are provided without combating silent disease.
\end{abstract}

Key words: Colorectal cancer, Colon, Rectum, Screening.

${ }^{1}$ Faculdades Santo Agostinho (FASA), Vitória da Conquista - BA. *E-mail: yasmin.felisberto@hotmail.com

SUBMETIDO EM: 3/2021

ACEITO EM: 4/2021

PUBLICADO EM: 4/2021 


\section{RESUMEN}

Objetivo: Revisar y reflexionar sobre la importancia de realizar pruebas de cribado de cáncer colorrectal (CCR). Revisión bibliográfica: CCR es una neoplasia que afecta al intestino grueso, siendo responsable de un número creciente de muertes en los países occidentales. En este escenario, en Brasil, con excepción del cáncer de piel no melanoma, esta neoplasia maligna tiene una alta incidencia, siendo el segundo tipo de cáncer más prevalente en mujeres y el tercero más prevalente en hombres. La mayoría de los tumores son esporádicos y pueden prevenirse mediante prevención primaria mediante una dieta equilibrada y actividad física regular. Además, en este contexto hay un foco en la prevención secundaria, ya que el uso de pruebas de cribado y campañas dirigidas a la identificación precoz son herramientas fundamentales para prevenir la progresión de la enfermedad tras el adenoma-carcinoma. Consideraciones finales: A pesar de la alta incidencia en el país, la CCR es una enfermedad curable cuando se detecta en etapas tempranas. Así, se desarrollan nuevos estudios sobre métodos de investigación, diagnóstico y cribado, se busca la superación profesional y se establecen políticas públicas eficientes de eficiencia que cubran la prevención secundaria sin combatir la enfermedad silenciosa.

Palabras clave: Cáncer colorrectal, Colon, Recto, Cribado.

\section{INTRODUÇÃO}

O organismo, para o seu bom funcionamento, necessita que todos os processos fisiológicos ocorram de forma harmônica e programada. Entretanto, alguns fatores comportamentais, ambientais e genéticos podem modificar esse ordenamento fazendo com que surjam as enfermidades. Nesse cenário, o crescimento celular é um evento normal e esperado, porém a proliferação rápida, incontrolável e agressiva gera transtornos orgânicos, como ocorre nas neoplasias. Assim, define-se como câncer um conjunto de mais de cem doenças que possuem características comuns no desenvolvimento e crescimento anormal de células que, além de desequilibrarem a homeostase, são capazes de formar tumores e invadir tecidos e órgãos vitais (INCA, 2018).

Com o passar dos anos, os avanços nos conhecimentos dentro do âmbito da tecnologia e ciência e o desenvolvimento socioeconômico foram fatores que contribuíram significativamente para melhorias na qualidade de vida dos indivíduos. Dessa forma, o crescimento e envelhecimento populacional é uma realidade que se associa diretamente com o aumento da incidência e prevalência de cânceres no mundo. Com isso, essa patologia torna-se um dos principais e mais desafiadores problemas de saúde pública enfrentados em diversos países, ocorrendo aproximadamente 18 milhões de novos casos por ano e metade desse número, infelizmente, evolui para óbito (BRAY F, et al., 2018).

Considerando o cenário brasileiro, estudos apontam que a cada ano surgirá cerca de 625 mil diagnósticos de câncer. Esses números foram estimados considerando o período de 2020 a 2022 e revelou, por ordem de incidência, os tipos que serão mais frequentes, sendo, respectivamente: o câncer de pele não melanoma, mama e próstata, colón e reto, pulmão e estômago. Do ponto de vista geográfico, a região Sudeste do país apresentará a maior porcentagem de casos, cerca de $60 \%$, seguidas das regiões Nordeste, com $27,8 \%$, e Sul, com 23,4\% (INCA, 2019).

Por ser uma enfermidade que afeta substancialmente as unidades formadoras dos seres vivos, o câncer pode se manifestar em qualquer sistema orgânico, atingindo estruturas que compõe, por exemplo, o intestino grosso dos indivíduos. Nesse contexto, surge então a denominação "Câncer Colorretal (CCR)" para as neoplasias que afetam o cólon, reto e ânus, podendo ainda ser delimitada de modo mais particular, conforme descreve a Classificação Internacional de Doença (CID-10), ao incluir nesses segmentos a junção retossigmóide (MENEZES CCC, et al., 2016).

Levando em consideração uma percepção global acerca do CCR, temos que atualmente esse tipo de patologia é considerada uma das mais prevalentes, principalmente, nas nações mais industrializadas. Logo, países da Europa, a Austrália, região norte do continente americano e o leste asiático, são alguns exemplos dos lugares que possuem os maiores números dessa neoplasia devido ao seu contexto histórico. Para mais, comparando o binômio "homem-mulher", estudos sugerem o sexo masculino como o grupo mundialmente 
mais acometido, estimando 1 milhão de novos casos por ano, ao passo que nas mulheres o câncer de cólon e reto se apresentam como o segundo tipo de tumor mais frequente, representando 800 mil novos casos (FERLAY J, et al., 2019).

O Instituto Nacional de Câncer José Alencar Gomes da Silva (INCA) é um importante órgão do Ministério da Saúde que, desde a sua fundação, busca promover informações atualizadas acerca dessa patologia. Assim, através de uma pesquisa de natureza descritiva a partir de inúmeros registros de hospitais brasileiros, ao longo de 38 anos, acredita-se que não há uma diferença exuberante entre a ocorrência do CCR entre homens e mulheres. Ambos os gêneros se encontram em faixas semelhantes, sendo os novos casos estimados apresentando um valor de 20.520 para o sexo masculino e 20.470 para o sexo feminino. Com isso, os riscos estimados a cada 100 mil habitantes também foram semelhantes, sendo, de 19,63 para homens e 19,03 para mulheres (INCA, 2019).

Grande parte dos tumores de cólon e reto originam-se de pólipos adenomatosos. Estes, por sua vez, surgem a partir de mutações nas células que integram a mucosa do intestino, podendo ocorrer com mais frequência em indivíduos inseridos em famílias com histórico para a doença. Inicialmente, essas estruturas anatômicas nada mais são que tumores benignos, porém, com o avançar da idade, podem adquirir o aspecto de malignidade. Nesse cenário, os programas e métodos de rastreamento tornam-se estratégias importantes para reduzir as complicações da doença, pois ao reconhecê-la precocemente o médico poderá promover tratamento eficaz e, consequentemente, um melhor prognóstico ao paciente (BRASIL, 2014; ASSIS RVBF, 2011).

Atualmente, o Ministério da Saúde recomenda o rastreamento do CCR na população com 50 a 75 anos de idade a partir do exame laboratorial (pesquisa de sangue oculto nas fezes) ou colonoscopias. Apesar da gravidade que essa doença pode adquirir e dos benefícios expressos que há na realização do diagnóstico precoce, não existe no país um programa populacional de rastreamento. Tal fato é justificado a partir dos resultados de ensaios clínicos que apontaram valores preditivos positivo baixos, alta porcentagens de falsos positivos e, por fim, inviabilidade do ponto de vista entre custo e benefício (BRASIL, 2010).

Diante dos dados mencionados, compreende-se, portanto, a relevância do tema, pois essa neoplasia é altamente incidente em diversas nações, revelando-se como um importante problema de saúde pública. Dessa forma, é necessário voltar os olhares da comunidade científica acerca da principal ferramenta que os profissionais de saúde podem utilizar para oferecer uma melhor qualidade de vida ao seu paciente. Com isso, essa pesquisa foi escrita e fundamentada no objetivo revisar e refletir acerca da importância da realização dos exames de rastreamento do Câncer Colorretal (CCR).

\section{REVISÃO BIBLIOGRÁFICA}

Considerada a terceira enfermidade maligna mais comum na população mundial, o CCR tem sua gênese compreendida em quatro estágios principais: iniciação, promoção, progressão e metástase. A primeira etapa é caracterizada por um dano genético irreversível que leva a célula à transformação neoplásica. Em seguida, as organizações celulares que sofreram alterações passam a se multiplicar promovendo um crescimento anormal e, caracterizando o terceiro estágio, podem sofrer novas modificações genéticas e epigenéticas. Nesse cenário, as células tumorais benignas passam a se tornar malignas, finalizando o processo com a sua disseminação através do sistema sanguíneo ou linfático para outros órgãos e tecido. Todo essa etiopatogenia leva décadas para que seja concluída (KEUM NN e GIOVANNUCCI E, 2019).

Entende-se por pólipos aquelas estruturas, com características neoplásicas ou não, que se projetam na superfície mucosa do organismo. Quando delimitamos o campo de estudo no sistema gastrointestinal, mais especificamente no intestino grosso, os pólipos terão um papel importante, pois são lesões precursoras para o início do CCR. Nesse caso, ocorrem alterações cromossômicas nas estruturas polipoides possibilitando sua transformação em adenomas, isto é, em lesões pré-malignas que ao longo do tempo, cerca de 10 a 15 anos, levam ao câncer. Os adenomas podem ser caracterizados como tubulares, o mais frequentemente encontrado, túbulo-vilosos e o viloso, sendo este o tipo que possui maior potencial para desenvolver malignidade (TORRES NETO JR, et al., 2010). 
Além do pólipo adenomatoso, existem os hiperplásicos. Entretanto, esse grupo não possui potencial para se tornarem lesões malignas. Normalmente, eles estão localizados distalmente no cólon e na área retal, desenvolvendo-se em indivíduos com idades mais jovens, o que não ocorre habitualmente nos adenomas convencionais (BICALHO LGMF, et al., 2015). Para mais, pode haver também uma lesão intermediária chamada de adenoma serrilhado. Esta é formada por tecido hiperplásico e adenomatoso que evolui para neoplasia. Com isso, percebe-se o quanto um estudo detalhado sobre os aspectos da origem biológica dessas estruturas é fundamental no contexto do CCR, pois a retirada dessas lesões e sua análise através do exame histopatológico é o que contribuirá para o diagnóstico definitivo (TORRES NETO JR, et al., 2010).

Os locais anatômicos mais acometidos do CCR são o segmento distal do reto, seguido pelo sigmoide, ceco, cólon ascendente e cólon transverso. Compreender essas regiões é fundamental, pois do ponto de vista clínico, os sinais e sintomas normalmente estão associados à localização do tumor, sendo a alteração do hábito intestinal a queixa mais prevalente entre os pacientes. Assim, realizar uma anamnse e exame físico individualizado e detalhado são as primeiras estratégias que o profissional de saúde irá utilizar para ter o CCR como hipótese diagnóstica, ainda mais no início dessa enfermidade. Afinal, o quadro clínico apenas se torna específico quando o indivíduo está numa fase mais avançadas da doença (MALLMANN GDP, et al., 2017).

Nesse contexto, percebe-se ainda que os tumores do reto apresentam como sinais clínicos o sangramento, muco e/ou pus, de forma isolada ou misturada nas fezes, acompanhado de tenesmo e sensação de evacuação incompleta. Por outro lado, os tumores do cólon direito estão mais associados com quadros de diarreia e síndrome dispéptica, contudo, nos estágios mais avançados, o paciente também pode evoluir com anemia e massa palpável no quadrante inferior direito. $O$ acometimento do cólon esquerdo promove obstipação intestinal progressiva, fezes escuras, com presença de sangue, em forma afiladas e períodos de alternância entre diarreia e constipação (VALADÃO M, et al., 2010).

Devido à característica de disseminação por via hematogênica ou linfática que as lesões malignas possuem, é interessante que o profissional de saúde possua uma visão ampliada do quadro clínico do paciente. Com isso, além dos sintomas discutidos anteriormente, deve ser alvo de investigação as queixas que incluem saciedade precoce, perda ponderal, anorexia, adenopatia na região supraclavicular e relato de sensação dolorosa em hipocôndrio direito. Indivíduos que se inserem nessas características são pacientes nos quais o médico deve ter uma atenção redobrada, pois sugere-se metástase distante ou contígua (MALLMANN GDP, et al., 2017).

Como a maioria das neoplasias, o CCR possui fatores de risco ambientais e genéticos. Em estudo, Bishehsari F, et al. (2014) relataram que o avanço socioeconômico em diversas populações influenciou na promoção dos hábitos de vida ocidentais e, consequentemente, o aumento desse câncer nos países considerados em desenvolvimento. Diante disso, destacam-se as mudanças nos hábitos de vida que incluem: dietas ricas em gorduras e carnes e geralmente pobre em cereais e fibras não refinadas; tabagismo; consumo excessivo de álcool e diminuição na prática habitual de atividades físicas. Somados a isso, há também o próprio envelhecimento populacional gerado pelo aumento da expectativa de vida.

Todavia, apesar de estar associada aos indivíduos de meia idade ou mais, o CCR também pode acometer grupos mais jovens. A origem dessa patologia nesse cenário pode ocorrer de forma casual ou por uma influência genética presente em famílias que possuem histórico desse câncer ou de outras desordens hereditárias, como é o caso da Síndrome de Lynch. Acredita-se que a combinação de variantes genéticas em diversos genes comuns de baixa penetrância e/ou a combinação destas com genes de alta penetrância característicos de um grupo familiar são alguns dos fatores que contribuem para o surgimento do CCR, sendo necessários mais estudos nesse âmbito para elucidar o processo fisiopatológico (STIGLIANO V, et al., 2014).

Partindo desse pressuposto, Parreiras FC, et al. (2013) afirmam que a maioria das lesões geradas pelo CCR são atribuídas por causas esporádicas e cerca de $70 \%$ dos casos ocorrem a partir dos 50 anos de idade. Porém, $10 \%$ dos pacientes diagnosticados se encontram inseridos dentre aqueles que desenvolveram a doença por predisposição hereditária. Os autores destacam a Polipose Adenomatosa Familiar, uma patologia hereditária autossômica dominante, sendo caracterizada por possuir inúmeros pólipos no cólon e possibilitar o desenvolvimento de câncer em $100 \%$ dos pacientes que não são submetidos a tratamento cirúrgico. 
Ademais, relatam patologias que podem acometer sítios diferentes daqueles que habitualmente estão associados ao CCR, como é o caso da Síndrome do Câncer Colorretal Hereditário sem Polipose (HNPCC).

Diante dos aspectos fisiopatológicos e das consequências, tanto físicas quanto psicossociais, que podem ser geradas no cotidiano das pessoas que convivem com esse tipo de câncer, a busca por ferramentas para auxiliar no diagnóstico precoce torna-se uma estratégia essencial para promover uma melhor qualidade de vida para esses pacientes. Logo, a adoção de métodos que proporcionem um rastreamento baseado na estratificação de risco individual aliados ao aprimoramento profissional, campanhas promovidas pelas políticas públicas e buscas ativas são medidas que interferem diretamente no aumento dos índices de cura (VIEIRA YZ, et al., 2013).

Um dos exames iniciais incluídos nos métodos de rastreio é a pesquisa laboratorial de sangue oculto nas fezes. O fundamento desse método é identificar a presença de hemoglobina, uma proteína presente nas células vermelhas do sangue, por meio de testes com guáiaco ou imunobiológicos. Este, em especial, recomenda-se a suspensão de aspirina e álcool por uma semana antes da realização do exame e não é indicado para mulheres durante o período menstrual ou pacientes que relatam queixas de pequena quantidade de sangue vermelho nas fezes ou ânus ou hematúria. Ademais, trata-se de um rastreio de baixo custo, podendo ser solicitado pelo profissional de saúde rotineiramente com o propósito de minimizar os gastos individuais ou públicos com medidas que abarcam a prevenção secundária (BRAGA DC, et al., 2017).

Embora ofereça algumas vantagens, a presença de sangue oculto nas fezes não é tão eficaz para definir o diagnóstico de CCR, sendo necessária a realização de exames complementares para compreender a origem do sangramento. Nessa perspectiva, o enema de duplo contraste é efetivo para visualizar pequenos pólipos, porém lesões planas que não provocam defeito de enchimento não são visualizadas. Além disso, se algo for encontrado não há como fazer a biópsia e retirada da lesão. Logo, a colonoscopia é o método mais adequado para rastrear e tratar os pólipos, contudo seu custo elevado e a possibilidade de complicações dificulta que esse exame seja utilizado de maneira rotineira no rastreamento (GUINHAZI NP, et al., 2019).

Para a população em geral, sem antecedente pessoal ou familiar, o rastreio do CCR deve ser recomendado aos 50 anos e continuado até os 75 anos. Em indivíduos assintomáticos e sem histórico que indique risco aumentado para a doença, deve-se iniciar o rastreamento com a pesquisa de sangue oculto nas fezes anualmente, acompanhado pela retossigmoidoscopia a cada dois a cinco anos. Os pacientes com alteração desses exames, devem complementá-los com colonoscopia. Quando possível, esta também pode ser feita como um exame de rastreio inicial a cada 10 anos quando não há alterações (FERRARI C, 2016).

Uma população que possua pouco conhecimento a respeito do CCR impede que haja redução dos fatores de risco e exames diagnósticos de rastreamento efetivos, contribuindo para maior incidência dessa neoplasia maligna. Um estudo norte-americano, feito pelo Center for Disease Control and Preventions Screen for Life mostrou que, após a realização de campanhas educativas sobre o câncer colorretal, a população demonstrou mais interesse em sua prevenção e, consequentemente, a mortalidade no país tem diminuído nos últimos anos (SANTOS TP, et al., 2013).

A partir de um estudo retrospectivo com pacientes da II Clínica Cirúrgica do Hospital Federal de Bonsucesso, Valadão M, et al. (2010) concluíram que o perfil dos indivíduos que buscavam tratamento naquela instituição refletia o cenário nacional e corroborava com as informações encontradas na literatura médica, pois a maioria dos 158 voluntários encontravam-se em estágio avançado do CCR. Ainda, relataram que $37 \%$ dos pacientes precisaram de cirurgias de emergência e mais de $70 \%$ estavam estratificados nos graus três e quatro de estadiamento. Para mais, criticaram o pouco acesso à informação que a população possui acerca dessa patologia, pois acreditam que se houvesse mais conhecimento os indivíduos iriam obter menos dificuldades em procurar assistência adequada, evitando o diagnóstico tardio.

No Brasil, o Sistema Único de Saúde (SUS) é uma vertente da Atenção Primária que oferece alternativas viáveis para auxiliar no processo de informação e recrutamento da população para iniciar o programa de rastreio pelo teste de sangue oculto nas fezes. Utilizando-se dos princípios de universalidade, integralidade e equidade no atendimento, as Estratégias de Saúde da Família podem utilizar a atuação do Agente 
Comunitário, Enfermeiro e Médico de família para realizar a busca ativa dos usuários daquela unidade através de telefonemas-convite, intervenções individuais e coletivas (SCANDIUZZ MCP, et al., 2019).

Quando indicado, o tratamento do CCR deve ser baseado na retirada dos pólipos através da colonoscopia. Para os minúsculos, isto é, aqueles menores que cinco milímetros, recomenda-se a excisão pela alça a frio ou biópsia de pinça. Por outro lado, pólipos considerados pequenos que estejam entre cinco a dez milímetros deve-se realizar um ressecamento por laço de polipectomia. Nessa perspectiva, os grandes pólipos, maiores de 20 milímetros, também podem ser ressecados durante a colonoscopia, salvo algumas exceções em que há necessidade de uma cirurgia laparoscópica. O mais importante é que o exame de colonoscopia seja de qualidade e que a remoção dessas lesões inclua uma margem de mucosa normal, de modo que não fique nenhuma lesão polipoide ou não polipóide com potencial cancerígeno (TRANQUILLINI CV, et al., 2018).

Após a realização da biópsia, a análise histológica da peça é feita para determinar o estadiamento do câncer. Logo, classificar a amostra em estágios nada mais é que definir a gravidade da doença para a condução de um tratamento mais eficaz e adequado. Para isso, utiliza-se o sistema TNM do American Joint Comittee on Cancer, no qual a letra T refere-se a extensão do câncer em camadas (1- mucosa, 2- submucosa, 3-muscular própria, 4- subserosa e serosa), a letra $\mathrm{N}$ informa se o câncer se espalhou pelo sistema linfático (varia de 1 a 3 , sendo que quanto maior o número, maior a quantidade de linfonodos acometidos) e a letra $\mathrm{M}$ que significa se há metástase ou não (se não utiliza-se o 0 e se sim utiliza-se o 1) (SOUZA GD, et al., 2018).

Em pacientes com diagnóstico tardio do CCR, quando há um elevado grau de acometimento do cólon, muitas vezes se faz necessário a realização da colostomia. Este procedimento cirúrgico, provisório ou definitivo, nada mais é que a exteriorização do cólon intestinal no abdome, precisamente na fossa ilíaca esquerda, e traz consigo dificuldades que ultrapassam o aspecto biológico da doença. Nesse cenário, existe a necessidade de se conviver com um estoma (do grego, se traduz em "abertura" ou "boca") e isso resulta na redução da qualidade de vida do paciente com neoplasia, pois implica constrangimentos físicos e psicossociais com sentimentos de dores e incertezas que necessitam da atuação de uma equipe multiprofissional para facilitar a aceitação, reabilitação e o cuidado do indivíduo e de sua família. (MACIEL DBV, et al., 2019)

O acompanhamento em doentes submetidos a ressecção do câncer deve ser realizado intensamente, afinal a recorrência da doença surge em aproximadamente $30 \%$ durante os primeiros dois anos após a cirurgia. Contudo, deve-se também dar uma atenção especial para o aspecto biopsicossocial do paciente, pois, conforme relatam Vieira LM, et al. (2013), a rotina exaustiva do tratamento, o pós-operatório e a convivência com a colostomia são fatores que, por necessitarem um enfrentamento tanto pessoal quanto social, podem gerar ansiedade, depressão, tristeza e isolamento. Dessa forma, além de se pensar nos programas de rastreamento como estratégias para evitar a progressão da doença, deve-se também ressaltar a importância da humanização dos profissionais de saúde a fim de amenizar esse sofrimento.

\section{CONSIDERAÇÕES FINAIS}

Por ser uma enfermidade que pode se manifestar em diferentes níveis de gravidade, o câncer é visto pelo olhar popular como uma doença que carrega indiretamente uma sentença de morte. Esse estigma, entretanto, deve ser enfrentado, pois os avanços tecnológicos e científicos contribuem para a construção de medidas preventivas e terapêuticas cada vez mais eficazes. Nesse contexto, o câncer colorretal deve ser avaliado como uma doença possível de ser combatida, afinal apresenta-se frequentemente de forma benigna, sendo curável quando detectado precocemente. Assim, desenvolver novos estudos sobre métodos de pesquisa que possam aprimorar o rastreamento e fortalecer as políticas públicas que incentivam a promoção desses programas são medidas essenciais para oferecer uma melhor qualidade de vida ao paciente.

\section{REFERÊNCIAS}

1. ASSIS RVBF. Rastreamento e Vigilância do Câncer Colorretal: Guidelines Mundiais. GED gastroenterol. endosc.dig., 2011; 30(2): 62-74. 
2. BICALHO LGMF, et al. Epidemiologia dos adenomas serrilhados em uma casuística do Hospital Alemão Oswaldo Cruz em São Paulo. GED gastroenterol. endosc. dig., 2015; 34(3):101-106.

3. BISHEHSARI F, et al. Epidemiological transition of colorectal cancer in developing countries: environmental factors, molecular pathways, and opportunities for prevention. World J Gastroenterol., 2014; 20(20): 6055-6072.

4. BRAGA DC, et al. Rastreamento do câncer colorretal através da pesquisa de sangue oculto fecal - um estudo de base populacional. GED gastroenterol. endosc. dig., 2017; 36(2): 60-64.

5. BRASIL. Caderno de Atenção Primária no 29. 2010. Disponível em: http://biblioteca.cofen.gov.br/wpcontent/uploads/2016/05/Cadernos-de-Aten\%C3\%A7\%C3\%A3o-Prim\%C3\%A1ria-n-29-rastreamento.pdf. Acessado em: 19 de março de 2020.

6. BRASIL. Protocolos clínicos e diretrizes terapêuticas em Oncologia. 2014. Disponível em: http://bvsms.saude.gov.br/bvs/publicacoes/protocolos_clinicos_diretrizes_terapeuticas_oncologia.pdf. Acessado em: 19 de março de 2020.

7. BRAY F, et al. Global cancer statistics 2018: GLOBOCAN estimates of incidence and mortality worldwide for 36 cancers in 185 countries. CA Cancer J Clin., 2018; 68(6): 394-424.

8. FERLAY J, et al. Estimating the global cancer incidence and mortality in 2018: GLOBOCAN sources and methods. International journal of cancer, 2019; 144(8): 1941-1953.

9. GUINHAZI NP, et al. Indicações e condutas de rastreamento de pólipos intestinais: uma revisão de literatura. Revista Artigos.Com, 2019; 1: e158.

10. INCA. ABC do câncer: abordagens básicas para 0 controle do câncer. 2018. https://www.inca.gov.br/sites/ufu.sti.inca.local/files//media/document//ivro-abc-6-edicao-2020.pdf. Acessado em: 19 de março de 2020.

11. INCA. Estimativa 2020: incidência de câncer no Brasil. 2019. Disponível em: https://www.inca.gov.br/sites/ufu.sti.inca.local/files//media/document//estimativa-2020-incidencia-de-cancer-nobrasil.pdf. Acessado em: 19 de março de 2020.

12. KEUM NN, GIOVANNUCCI E. Global burden of colorectal cancer: emerging trends, risk factors and prevention strategies. Nature Reviews Gastroenterology and Hepatology, 2019; 16(12): 713-732.

13. MACIEL DBV, et al. Perfil sociodemográfico de pacientes com estomia definitiva por câncer colorretal: Interferência na qualidade de vida. Nursing, 2019; 22(258): 3339-3344.

14. MALLMANN GDP, et al. Câncer colorretal. Acta méd., 2017; 38(7): 1-7.

15. MENEZES CCC, et al. Câncer colorretal na população brasileira: taxa de mortalidade no período de 2005-2015. Revista Brasileira em promoção da Saúde, 2016; 29(2): 172-179.

16. PARREIRAS FC, et al. Genetic aspects of colorectal cancer and its impact on disease management. Revista Médica de Minas Gerais, 2013; 23(2): 221-227.

17. TRANQUILLINI CV, et al. Revisão sistemática e meta-análise sobre técnicas de polipectomia de pólipos colorretais pequenos e diminutos. Arq. Gastroenterol., 2018; 55(4): 358-368.

18. SCANDIUZZ MCP, et al. Câncer Colorretal No Brasil: Perspectivas Para Detecção Precoce. Brasília Med., 2019; 56: 8-13.

19. SANTOS TP, et al. Conhecimento dos usuários do serviço público de saúde sobre câncer colorretal e sua prevenção. Rev. AMRIGS, 2013; 57(1): 31-38.

20. SOUZA GD, et al. Métodos De Imagem No Estadiamento Pré E Pós-operatórios Do Câncer Colorretal. ABCD, arq. bras. cir. dig., 2018; 31(2): e1371.

21. STIGLIANO V, et al. Early-onset colorectal cancer: a sporadic or inherited disease? World J Gastroenterol., 2014; 20(35):12420-30.

22. TORRES NETO JR, et al. Aspectos epidemiológicos dos pólipos e lesões plano-elevadas colorretais. Rev bras. coloproctol., 2010; 30(4): 419-429.

23. VALADÃO M, et al. Perfil dos pacientes portadores de câncer colorretal operados em um hospital geral: necessitamos de um programa de rastreamento acessível e efetivo. Rev bras. colo-proctol., 2010; 30(2): 160-166.

24. VIEIRA LM, et al. Câncer Colorretal: entre o sofrimento e o repensar na vida. Saúde em Debate, 2013; 37(97): 261 269.

25. VIEIRA YZ, et al. Rastreamento e diagnóstico precoce de câncer colorretal / Screening and early diagnosis of colorretal câncer. Acta méd., 2013; 34(5): 1-5. 